\title{
Development of a framework for increasing asthma awareness in Chitungwiza, Zimbabwe
}

\author{
Pisirai Ndarukwa ${ }^{1 *}$, Moses J. Chimbari ${ }^{1}$ and Elopy N. Sibanda ${ }^{2}$
}

\begin{abstract}
Background: Asthma accounts for significant global morbidity and health-care costs. It is still poorly understood among health professionals and the general population. Consequently, there are significant morbidity and mortality rates throughout the globe. The aim of this study was to develop a framework to increase asthma awareness at Chitungwiza Hospital, Zimbabwe.

Methods: A modified Delphi model was used to collect data to develop a framework for increasing asthma awareness. At baseline (round 1) in-depth interviews with 44 medical doctors were carried out to understand the level of asthma awareness. Round 2 data collection was in the form of a workshop involving a total of 15 doctors, 30 nurses, four public relations officers, and two health education and promotion officers. The same participants who were engaged in round 2 were also involved in the third round where consensus was achieved by the health professionals.

Results: Our study showed that awareness to asthma among health care providers was affected by mimicry of symptoms and lack of continuous education on asthma. Our study showed lack of Information Education and Communication (IEC) material and lack of use of bulk messages affected asthma awareness. Our study showed that clinical meetings on asthma, having asthma training manuals, (IEC) materials and guidelines for asthma diagnosis and management could improve health care providers' awareness of asthma. Bulk messages on asthma through network providers, social media and bill boards, commemorating world asthma day, edutainment, asthma ambassadors and multimedia were suggested as means of improving awareness of asthma among the public.
\end{abstract}

Conclusion: We concluded that awareness of asthma can be improved using a framework. Such a framework ultimately improves the quality of asthma care.

Keywords: Asthma, Asthma awareness, Framework, Chitungwiza, Zimbabwe

\section{Background}

Asthma accounts for significant global morbidity and health-care costs. It is defined as a chronic inflammatory disorder of the small airways whose pathogenesis is linked to variable structural changes that affect both children and adults of all ages [1] Allergic asthma is associated with inhalation of an allergen that stimulate immune response [2].. Despite the high global mortality rate due to asthma, it is still not fully understood among health professionals and the general population [3].

\footnotetext{
* Correspondence: papandarukwa@gmail.com

${ }^{1}$ College of Health Sciences, School of Nursing and Public Health, University of KwaZulu Natal, 1st Floor, George Campbell Building, Howard College Campus, UKZN, Durban 4000, South Africa

Full list of author information is available at the end of the article
}

Health messages that reach the entire spectrum of stakeholders at once have been reported to raise awareness of patients, caregivers and health facility managers and policymakers [4]. However, to our knowledge there are no frameworks to ensure that the health messages for asthma reach the health care workforce and patients. Knowledge of asthma symptoms and signs enhances the ability of the health care workers and patients to take appropriate health care decisions [5]. It has been reported that effective asthma control in patients and the population at large depends on their ability to recognise the range of factors influencing the management of the disease [6].

A review of strategies for strengthening asthma education to improve disease control by Clark, et al. [5], reported that

(C) The Author(s). 2019 Open Access This article is distributed under the terms of the Creative Commons Attribution 4.0 International License (http://creativecommons.org/licenses/by/4.0/), which permits unrestricted use, distribution, and 
family, clinicians, friends, neighbours and work or school mates in the patient's social environment could act as barriers to effective asthma control due to lack of knowledge about the disease. The health care providers were reported to lack ability to effectively identify asthma cases and educate patients on asthma control [7].

Asthma management techniques including patient education were found not to be sensitive or relevant to culture and language thus causing some difficulties in some ethno cultural communities and minority groups [8]. Therefore, there is need to design a framework which is culturally and language sensitive in order to improve knowledge across different groups of people (patients, communities and health professionals) about asthma [9].

Rosas-Salazar, et al. [10], showed that poor health literacy is an important barrier to asthma knowledge among children and adults. Another study in Zambia showed that only a few health workers were trained in asthma management and they still could not clearly identify asthma cases [11]. Another study in South Africa revealed that health care workers could not distinguish between asthma and chronic obstructive pulmonary disease [12]. This indicates the importance of improving awareness and knowledge of asthma among health care workers for better management of the disease.

Apathy for participation in refresher asthma management courses targeting health care workers has been cited as a contributory factor for poor asthma management [13]. Most health workers are unaware of and do not follow the international guideline on asthma management [13]. This is indicative of the knowledge gap on asthma that exists among health workers. Hence, the need for establishing an asthma awareness framework that may help practitioners and the general public to have more knowledge on asthma management.
The aim of this study was to develop a framework for asthma awareness to improve the ability to recognise asthma within the population and amongst health care providers. The framework is intended to enhance the general public's knowledge about asthma to make them pro-active in seeking health care for asthma. For health care workers the framework will improve their ability to find cases through appropriate diagnosis informed by the framework.

\section{Methods \\ Study design}

We used the modified three stage Delphi method to develop an awareness framework for asthma $[14,15]$. The modified process involved three rounds of data collection intended to distil knowledge and build reliable consensus regarding an awareness framework for asthma among health care providers and the public. Figure 1 presents a flowchart of the participants of the three rounds. All the first round participants (doctors) were invited to participate in the final two rounds. In addition, nurses, public relations officers, health education and health promotion officers and the medical experts (specialist doctors) who formed the panel experts were added for rounds 2 and 3 (Fig. 1).

\section{Round 1}

We aimed to obtain all potentially significant items for the asthma awareness framework. We conducted indepth interviews with key informants (doctors who were in general practice in medicine and specialist doctors in medicine involved in asthma care). The participants included 41 general doctors and 3 specialist physicians who were based at Chitungwiza Central Hospital. These doctors were briefed on the study aim and objectives.

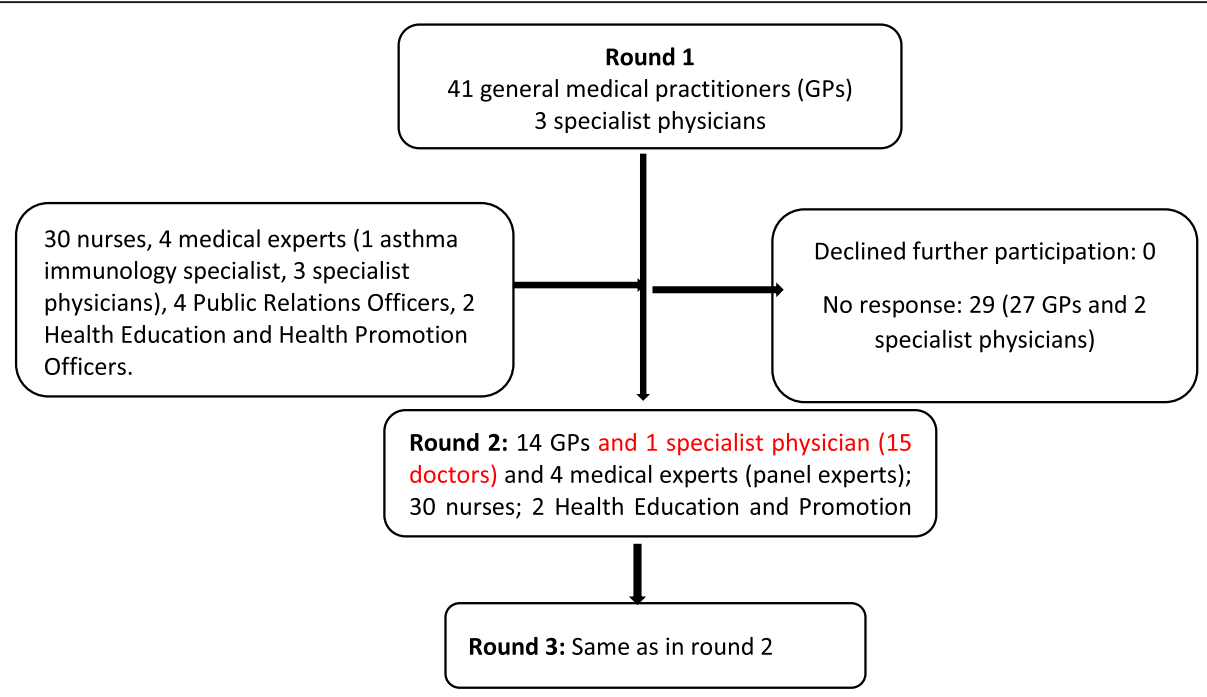

Fig. 1 Flowchart of the process of Delphi model showing study participants at each round of participation 
Consent was sought from them to participate in a face to face interviews. The interview solicited for information on the perceived definition and clinical presentation of asthma, difficulties they faced in asthma diagnosis and management; and challenges which they thought were experienced by the health care team and public with regards management of asthma.

\section{Round 2}

Fifteen doctors and 30 nurses who worked in the medical departments at the hospital, 2 health promotion and education officers (1 working at Chitungwiza Central Hospital $(\mathrm{CCH})$ and the other one working for Chitungwiza City Health Department (CCHD)); and 4 public relations officers (3 working at $\mathrm{CCH}$ and 1 working at $\mathrm{CCHD}$ ) participated in the round 2 data collection phase. This was done through a workshop convened as a follow-up to the first round of data collection. This workshop was led and facilitated by medical experts in asthma care.

Participants at the workshop were divided into two; Group 1 focused on the asthma awareness among the health care providers and Group 2 focused on the asthma awareness among the public.

\section{Round 3}

In this round of data collection, a panel of medical experts led the process. The two groups were asked to present information on what they believed should be included in the framework for asthma awareness targeting the health care providers and public. Each group presented and a plenary discussion moderated by the experts followed. The objective of the discussion was to modify and adopt information on group assignments (see Fig. 1).

\section{Data analysis}

Data collected in round 1 using in-depth interviews was constructed into consensus statements. The subsequent rounds were done to solidify the presence of consensus. Research findings were reported in thematic format.

\section{Results}

\section{Round 1 results}

In the initial round of the study, results of the in-depth interviews resulted in three thematic areas which were (i) lack of awareness of asthma among some healthcare providers, (ii) inability to diagnose asthma, and (iii) ways to enhance awareness of asthma. These themes are summarized in (Additional file 1: Table S1).

\section{Theme 1: asthma awareness among health care providers}

The respondents interviewed in this phase indicated there was a general lack of awareness of asthma and it's symptoms among healthcare providers. Some of the sentiments expressed by the interviewees are indicated in
Table 1. Results indicated that respondents felt that health care providers lacked knowledge of asthma presentation, and were inexperienced especially in relation to the ability to tie up presenting complaints and the history that patients provided.

Asthma awareness among patients and the general public Our study revealed that interview respondents concurred on the need for creating awareness for asthma among the public through the use of bill boards, bulk messages that are transmitted through network providers, use of asthma champions, social media, commemorations of world asthma days and use of multimedia (Table 1). Healthcare providers highlighted the need to inform the public of the risk factors for asthma such as history of allergies and atopy, and family history of asthma. The doctors indicated the need for symptoms of asthma to be packaged IEC materials which would be widely distributed into the communities as a way of increasing awareness of asthma.

\section{Theme 2: inability to diagnose asthma}

Results from the in-depth interviews revealed healthcare providers were having difficulties diagnosing asthma (Table 1) and this was mainly due to lack of diagnostic equipment such as Peak expiratory flow meters, spirometers, reagents for RAST tests, Bronchial provocation tests and non-availability of definitive lung function for asthma diagnosis. Additionally, clinicians reported that the presence of comorbidities such as emphysema and COPD posed challenges to some healthcare providers when diagnosing asthma. Some respondents reported having difficulties in differentiating asthma from heart diseases which affected their clinical judgement relating to asthma diagnosis.

\section{Theme 3: ways to enhance awareness}

Respondents suggested a number of ways to create and improve awareness of asthma (see Table 1). Interviewees emphasized on the need for an awareness framework for health care providers and for the public. Also raised during the interviews was the need for current asthma algorithms for diagnosis in the consultation rooms for health care providers as a way of creating and enhancing asthma awareness among clinicians. There was consensus among all doctors who said clinical meetings would improve awareness of asthma. They also highlighted the need for constant workshops on recent developments on asthma diagnosis and management. Communication with other health care workers involved in asthma diagnosis and management such as nurses was also important. 


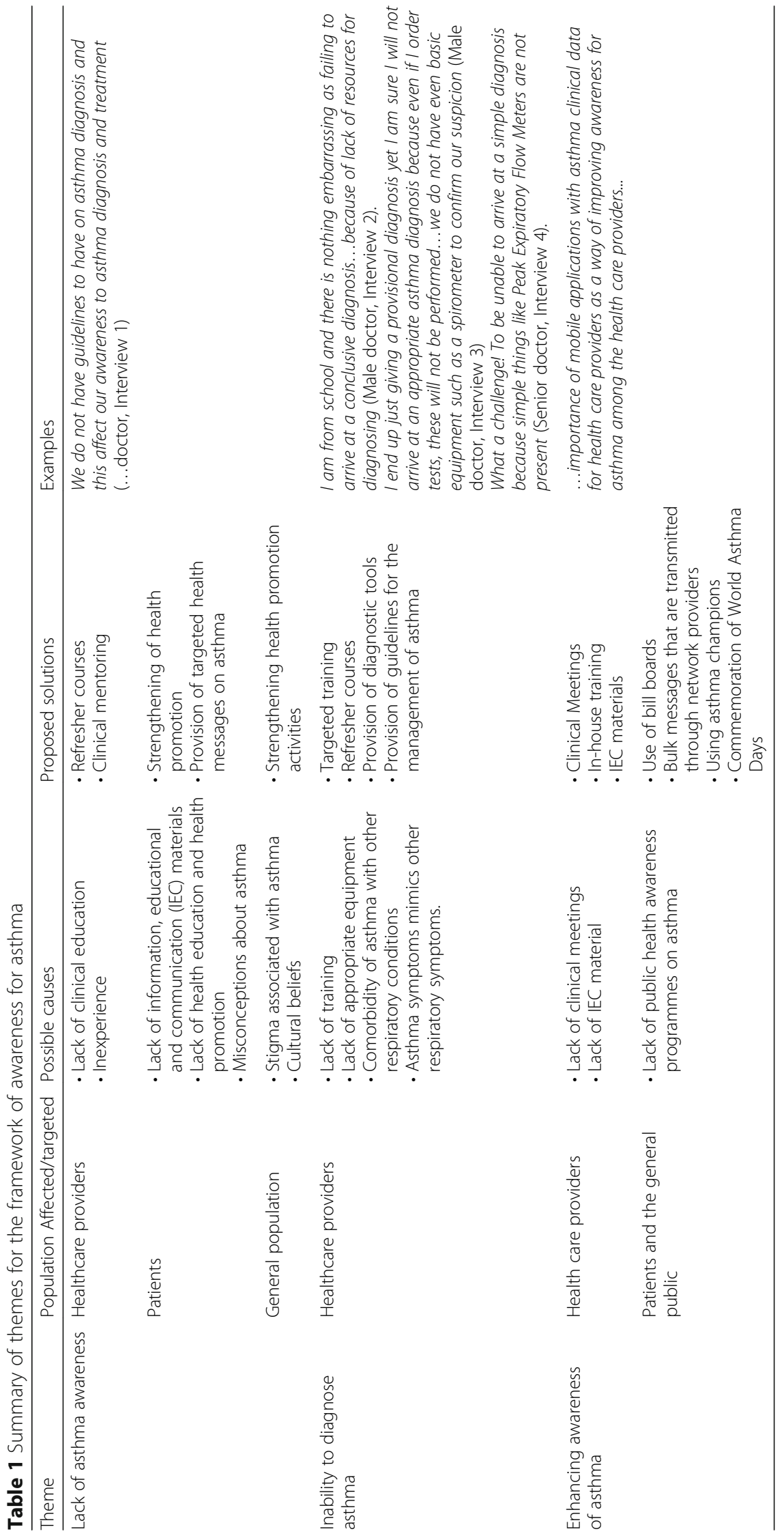


Round 2 results

The themes emerged from round one of the study were presented to respondents during round 2 . Results indicated that the viewpoints from round 2 were not at variance with the initial in-depth interviews which had been conducted with the doctors. The panel of experts who moderated during the presentation, asked participants how they expected the awareness framework to look like. All participants agreed that the asthma awareness framework should benefit both the health care professionals and the public.

They suggested that asthma awareness framework should be implemented through; Use of Clinical workshops (health professional), Training manuals, and Information Education and Communication (IEC) materials for consultation rooms (Posters). Bulk messages through mobile telecommunications network providers such as NetOne, Telecel and Econet. Use of champions (Asthma ambassadors such as popular musicians), Social media, Bill boards, Commemorations of world asthma day (take note of seasonality issues), (Ministries of health, environment), edutainment (road shows, dramas), Multimedia (Bill boards, Televisions and radio adverts on asthma, Pamphlets).
Round 3

A draft framework was developed by the authors which was then presented to the panel of experts for adoption. The participants adopted the asthma awareness framework (Fig. 2).

\section{Discussion}

Using the modified Delphi model (in-depth interviews, group works and group presentations, and panel discussion) we developed an asthma awareness framework. The study consisted of three phases leading to consensus on the asthma awareness framework. All the participants for all phases suggested that clinical meetings on asthma, having asthma training manuals, IEC materials and guidelines for asthma diagnosis and management could improve awareness of asthma by health care providers. It was also suggested that bulk messages on asthma through network providers, social media and bill boards, commemorating world asthma day, edutainment, asthma ambassadors and multimedia may improve awareness of asthma among the public. We concluded that efforts to improve awareness for asthma should involve all the stakeholders.

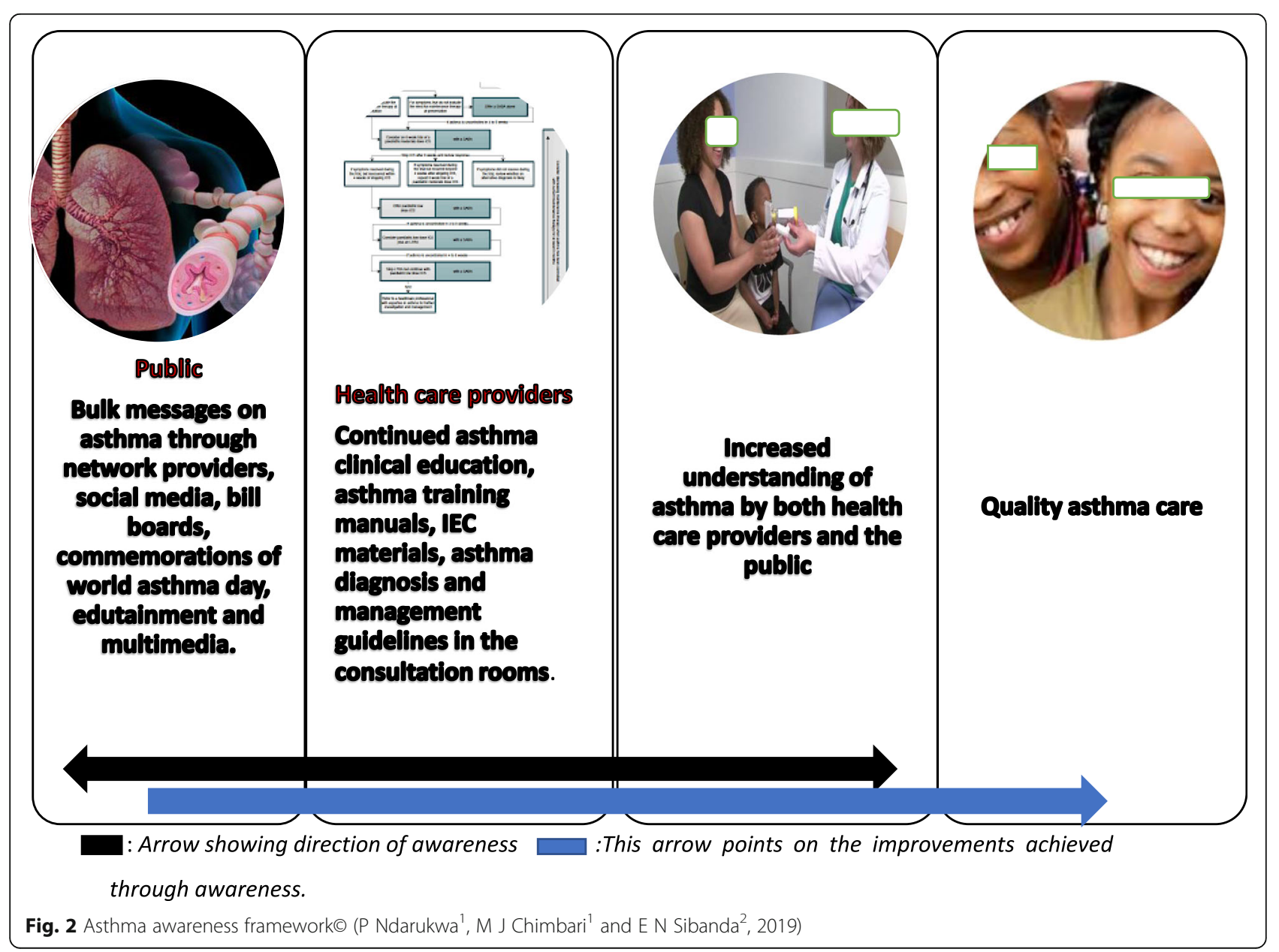




\section{Awareness of asthma among health care providers}

Holding of the clinical meetings on asthma and having training manuals were suggested as means for improving awareness of asthma among health care professional. These strategies address the lack of asthma knowledge by care providers who may experience difficulties in diagnosing and managing asthma. Our study findings are in agreement with other studies [16-18] that have underscored the importance of education sessions to improve the awareness of asthma. Kang, et al. [18], reported that effective educational tools are important for increasing adherence to asthma guidelines and clinical improvement of asthma patients.

Information, Education and Communication materials and guidelines for asthma diagnosis and management were also reported in our study as some of the strategies to improve awareness of health care professions in asthma. Other studies have also shown that IEC improves people's health by increasing their awareness and knowledge; and changing attitudes and behaviours [1921]. Furthermore, guidelines for asthma diagnosis and management are reported as effective means by which health care professionals can learn rapidly and amass vast knowledge and skills in asthma, thus contributing to their awareness of the condition [21].

\section{Awareness of asthma among the public}

This study has indicated that the public's awareness of asthma can be increased by use of bulk messages through network providers. Sharing asthma messages is a key component towards achieving asthma awareness and this promote the community to be proactive in dealing with cases of asthma [22]. The use of network providers can rapidly spread messages about asthma ensuring that a wide audience is reached at one goal thus improving awareness. Bill boards were also reported in our study to be effective means for public awareness of asthma. This has been revealed in other studies that reported bill boards as effective tools for mass awareness [23, 24]. Other studies have suggested that messages on the bill board must be displayed with pictures that can easily be comprehended by the general public $[23,25]$.

In this study we have learnt that multimedia can improve asthma awareness to the public. This was also reported in other studies that demonstrated that local news media can be an effective partner for disseminating asthma messages [26, 27]. Use of local television and radio-talk shows hosting asthma care professionals to address community asthma awareness, thus improving understanding the public about asthma [26]. Our study further asserted that edutainment in the form of drama and role plays can improve asthma awareness among the public. This is consistent with findings from other studies that have reported extensively on the role of edutainment [28-30].

Social media was suggested, in our study, as important for improving asthma awareness. This is also reflected in another study indicating that social media is increasingly being used as a tool to disseminate information on diseases to increases public awareness [31].

Consistent with the World asthma report [31, 32], our study suggests that commemoration of world asthma day can improve asthma awareness among the public.

\section{Conclusion}

We developed a framework for asthma awareness appropriate for health care providers and the public. We recommend the adoption of the framework for asthma awareness of the Ministry of Health and Child Care, Zimbabwe.

\section{Supplementary information}

Supplementary information accompanies this paper at https://doi.org/10. 1186/s40733-019-0052-2

Additional file 1: Table S1. Summary of themes for the framework of awareness for asthma.

\section{Abbreviations}

CCH: Chitungwiza Central Hospital; CCHD: Chitungwiza City Health Department; COPD: Chronic Obstructive Pulmonary Disease; GPs: General Practitioners; IEC: Information, Education and Communication; PEF: Peak Expiratory Flow; RAST: Radioallergosorbent testing; UKZN: University of KwaZulu-Natal

\section{Acknowledgements}

This study received funding from the OAK foundation and UKZN College of Health Sciences to ensure that the PN was able to do field data collection and analysis of data. PN is an OAK foundation fellow.

We would like to acknowledge Professor Mujuru, Professor Mukaratwira, Dr. Chitiyo, Nurses, Public relations officers and health promotions and health education officers who contributed in during the data collection workshop.

\section{Authors' contributions}

PN and MJC conceived the idea. PN with the assistance of MJM designed the protocol and revisions were done by MJC and ENS. PN designed the protocol. PN wrote the first draft of the manuscript and all authors (PN, MJC and ENS) reviewed changes. All authors read and approved the manuscript for submission to the journal.

\section{Funding}

This study was funded by OAK foundation and UKZN College of Health Sciences to ensure that the PN was able to do field data collection and analysis of data.

\section{Availability of data and materials}

The datasets used and/or analysed during the current study are available from the corresponding author on reasonable request.

\section{Ethics approval and consent to participate}

We obtained ethics approval to conduct the study from Medical Research Council of Zimbabwe (A/2352) and Biomedical Research Ethics Committee of the University of KwaZulu-Natal (BE613/18). Gatekeepers' permission was sought from Chitungwiza Hospital as well as Ministry of Health and Child Care, Zimbabwe. 


\section{Competing interests}

The authors declare that they have no competing interests.

\section{Author details}

${ }^{1}$ College of Health Sciences, School of Nursing and Public Health, University of KwaZulu Natal, 1st Floor, George Campbell Building, Howard College Campus, UKZN, Durban 4000, South Africa. ${ }^{2}$ Asthma, Allergy and Immune Dsyfunction Clinic, 113 Kwame Nkrumah Ave, Harare, Zimbabwe.

Received: 17 May 2019 Accepted: 10 October 2019

Published online: 29 October 2019

\section{References}

1. Pavord ID, et al. After asthma: redefining airways diseases. Lancet. 2018; 391(10118):350-400

2. Holgate ST. Innate and adaptive immune responses in asthma. Nat Med 2012;18:673.

3. Bousquet J, et al. Allergic rhinitis and its impact on asthma (ARIA). Allergy. 2008;63:8-160

4. Smith SL, Shiffman J. Setting the global health agenda: the influence of advocates and ideas on political priority for maternal and newborn survival. Soc Sci Med. 2016;166:86-93.

5. Clark NM, Partridge MR. Strengthening asthma education to enhance disease control. Chest. 2002;121(5):1661-9.

6. Canino $G$, et al. Addressing asthma health disparities: a multilevel challenge. J Allergy Clin Immunol. 2009;123(6):1209-17.

7. Clark NM. J.A.r.o.p.hCommunity-based approaches to controlling childhood asthma. Annu Rev Public Health. 2012;33:193-208.

8. Ghanname I, et al. Factors associated with asthma control: MOSAR study (Multicenter Observational Study of Asthma in Rabat-Morocco). BMC Pulm Med. 2018;18(1):61.

9. Poureslami IM, et al. A systematic review of asthma and health literacy: a cultural-ethnic perspective in Canada. MedGenMed. 2007;9(3):40.

10. Rosas-Salazar C, et al. Health literacy and asthma. J Allergy Clin Immunol. 2012;129(4):935-42.

11. Somwe Wa S, et al. Improving paediatric asthma care in Zambia. Bull World Health Org Suppl. 2015;93(10):732-6.

12. Mash B, Rhode H, Pather M, Ainslie G, Irusen E, Bheekie A, Mayers P. Quality of asthma care: western Cape province, South Africa. S Afr Med J. 2009; 99(12):892-96.

13. Fawibe $A E$, et al. Drug prescription pattern for asthma among nigerian doctors in general practice: a cross-sectional survey. Annals of thoracic medicine. 2012;7(2):78-83.

14. Okoli C, Pawlowski SD. The Delphi method as a research tool: an example, design considerations and applications. Inf Manag. 2004;42(1):15-29.

15. Murphy MK. Consensus development methods, and their use in clinical guideline development. Health Technol Assess. 1998;2(3):1-88.

16. Kavut $A B$, Kalpakloğlu AF. Impact of asthma education meeting on asthma control level assessed by asthma control test. The World Allergy Org J. 2010;3(1):6-8.

17. Wa Somwe S, et al. Improving paediatric asthma care in Zambia. Bull World Health Org Suppl. 2015;93:732-6.

18. Kang S-Y, et al. The virtual asthma guideline e-learning program: learning effectiveness and user satisfaction. Korean J Intern Med. 2018;33(3):604-11.

19. Welfare, M.o.H.a.C., IEC Reference manual for health programme managers. 1998.

20. Gawde NC, Sivakami M, Babu BV. Building partnership to improve Migrants' access to healthcare in Mumbai. Front Public Health. 2015;3:255.

21. Global Initiative for Asthma. 2018 GINA Report, Global Strategy for Asthma Management and Prevention. 2017

22. Rhee $\mathrm{H}$, et al. Mobile phone-based asthma self-management aid for adolescents (mASMAA): a feasibility study. Patient Prefer Adherence. 2014;8:63.

23. Noar SM. A 10-year retrospective of research in health mass media campaigns: where do we go from Here? J Health Commun. 2006;11(1):21-42.

24. Antic R, et al. "Could it be asthma?": the impact of a mass media campaign aimed at raising awareness about asthma in Australia. Health Educ Res. 1993;8(4):581-7.

25. Evers $U$, et al. Asthma in older adults: the need for asthma health promotion interventions. J Asthma Allergy Educ. 2013;4(4):183-90.

26. Wright RJ, Fisher EBJN. Putting asthma into context: community influences on risk, behavior, and intervention. Neighborhoods and health. 2003:233-64.

27. Homes H. National Health Education Week; 2007.
28. Luna-Nevarez C, et al. Transforming health care: empowering therapeutic communities through technology-enhanced narratives. J Consum Res. 2014 41(2):237-60.

29. Papa MJ, Singhal A. How entertainment-education Programmes promote dialogue in support of social change. J Creat Commun. 2009;4(3):185-208.

30. Wiemeyer J, Tremper LL. In: Nakatsu R, Rauterberg M, Ciancarini P, editors. Edutainment in Sport and Health, in Handbook of Digital Games and Entertainment Technologies. Singapore: Springer; 2017. p. 883-908.

31. Mydin HH, Ampikaipakan S, Shayaa S, Partridge MR. The effect of an asthma awareness campaign on social media users. Eur Respir J. 2018;52(Suppl 62): PA3137.

\section{Publisher's Note}

Springer Nature remains neutral with regard to jurisdictional claims in published maps and institutional affiliations.
Ready to submit your research? Choose BMC and benefit from:

- fast, convenient online submission

- thorough peer review by experienced researchers in your field

- rapid publication on acceptance

- support for research data, including large and complex data types

- gold Open Access which fosters wider collaboration and increased citations

- maximum visibility for your research: over $100 \mathrm{M}$ website views per year

At BMC, research is always in progress.

Learn more biomedcentral.com/submissions 